\title{
DESARROLLO DE UN SISTEMA DE NEUROMARKETING USANDO EL DISPOSITIVO EMOTIV-EPOC
}

\section{DEVELOPMENT OF NEUROMARKETING SYSTEM USING EMOTIV-EPOC DEVICE}

\section{RESUMEN}

Este artículo presenta los avances concebidos en la realización de un sistema de neuromercadotecnia. El objetivo del sistema es permitir evaluar las emociones que presentan los televidentes al observar comerciales publicitarios. Se hace uso de la interfaz cerebro-computador Emotiv-EPOC para la adquisición de las neuroseñales. Se describen los algoritmos empleados para el análisis de las pautas publicitarias. Se presentan algunas pruebas experimentales donde se comparan los resultados obtenidos mediante el sistema propuesto, con respecto a los métodos tradicionales.

Palabras clave: mercadotecnia, neurociencia, procesamiento de señales.

\begin{abstract}
This paper presents some advances obtained by the development of neuro-marketing systems. The aim of the systems is allow assessment of emotions that generates commercial advertising over viewers. The brain-computer interface Emotiv-EPOC was used for neuro-signals acquisition. The algorithms used for analysis of the advertising patterns are described. Some experimental tests where the results obtained by traditional methods are compared with the proposals are presented.
\end{abstract}

Keywords: marketing, neuroscience, signal processing.

\section{Luz Ángela Moreno}

Magister en Dirección y Administración de Empresas, administradora comerial y de sistemas

Universidad de Pamplona

luzamoreno02@gmail.com

Pamplona, Colombia

\section{César Augusto Peña}

Doctor en Automática y Robótica Docente Universidad de Pamplona cesarapc@unipamplona.edu.co Pamplona, Colombia

\section{Oscar Eduardo Gualdrón}

Doctor en Electrónica

Docente Universidad de Pamplona oscar.gualdron@unipamplona.edu.co Pamplona, Colombia

Tipo: Artículo de investigación

Fecha de Recepción: Junio 25 de 2014

Fecha de Aceptación: Octubre 20 de 2014 


\section{INTRODUCCIÓN}

Hoy en día la competencia que se evidencia en el mercado es significativa, lo que impulsa a las empresas a realizar cada vez mayores esfuerzos para poder cautivar la atención del público o de los futuros consumidores finales. Es por ello que se hace necesario conocer o incluso generar necesidades y deseos en los posibles usuarios, por lo que las empresas deben hacer uso de las diversas herramientas, procesos, tácticas y técnicas que logren cautivar la atención del cliente. Este tipo de actividades hacen parte del denominado marketing.

El marketing es una disciplina que se ha ido desarrollando desde el siglo pasado. Esta palabra ha sido adoptada y utilizada por las distintas instituciones de los ámbitos tanto académico como laboral, debido a que la definición de ella en español ("mercadotecnia", según la Real Academia Española) no cubre en su totalidad su significado, por tanto, para efectos de este artículo se hará mención a la palabra marketing.

Son múltiples los autores, organizaciones e instituciones que han venido desarrollando este concepto. Las definiciones que mayor peso tienen son las dadas por la Asociación Americana de Marketing (AMA) [1] y la de Philip Kotler, considerado el padre del marketing moderno (según el Centro Europeo de Administración [2]), por sus diversas obras, como Fundamentos del marketing, Dirección de marketing, Innovar para ganar, Principios de marketing, Caótica, entre otros.

El marketing es una disciplina encargada de analizar el comportamiento de los mercados y de los consumidores. Es asumida por las empresas que desean incrementar sus beneficios económicos, cautivando nuevos clientes y reforzando la fidelidad de los ya existentes. Esto se realiza por medio del empleo de diversas técnicas y tácticas que permitan detectar las necesidades de los clientes de forma más efectiva o incluso generar deseos por un producto especifico.

Anteriormente existía poca competencia, y las empresas para obtener una ventaja competitiva buscaban conocer más a través de encuestas y cuestionarios los deseos, gustos y necesidades de los consumidores con el fin de satisfacerlos, además de obtener beneficios económicos. Pero desde hace un tiempo se ha evidenciado que esto no es suficiente para conocer sobre las decisiones que tomará determinado usuario respecto a un producto específico. Por ello se han desarrollado diferentes técnicas con el fin de evaluar los comportamientos que pueden tener las personas de acuerdo con las emociones [3], el grado de atención [4], las expresiones corporales, entre otros [5].

Entre las técnicas actuales sobresalen algunas que emplean equipos como pulsómetros o electrocardiógrafos para medir la frecuencia o ritmo cardiacos [6], los GSR (galvanic skin response), que permiten medir la resistencia galvánica de la piel, la cual cambia [7] debido a la activación de las glándulas sudoríparas al generarse sentimientos de miedo, ira o sexuales; el electromiógrafo, que permite registrar la actividad eléctrica de músculos [8], detectores o seguidores del movimiento de los ojos [9], unidades inerciales para detectar movimientos bruscos de los usuarios, polisomnógrafos para medir los cambios respiratorios, y uno de los más recientes: los electroencefalógrafos (ECG), que permiten registrar la actividad bioeléctrica del cerebro [10].

Este último instrumento es uno de los pilares para el estudio y la investigación de los estímulos conscientes y subconscientes de los usuarios, que permiten analizar las conductas, decisiones y proceso mentales de los mismos en los campos de acción del marketing. El empleo de estas técnicas origina el denominado neuromarketing. Adicionalmente, en esta disciplina se pueden utilizar información cerebral como la dada por la resonancia magnética funcional (fMRI) [11], la magnetoencefalografía (MEG) y la tomografía de emisión de positrones (PET) [12].

El sistema propuesto en este artículo se basa en el uso de un dispositivo denominado EmotivEPOC que procesa las señales encefalográficas 
o neuroseñales y permite un análisis cognitivo, la detección de expresiones faciales y un análisis de emociones. Es un dispositivo de bajo costo y de fácil adquisición. La mayoría de sus aplicaciones se presenta como una interfaz cerebro-computador $(\mathrm{BCI})$ para controlar diversos software [13].

La aplicación propuesta en este artículo, para la interfaz se presentará de la siguiente forma: en la sección dos se hace una descripción del dispositivo que captura las neuroseñales; en la sección 3 se plantea el algoritmo empleado para el análisis de las pautas publicitarias (spot); en la sección 4 se analizan los resultados obtenidos y por último se presentas las conclusiones.

\section{DISPOSITIVO DE CAPTURA DE SEÑALES CEREBRALES}

El cerebro es el principal órgano del sistema nervioso central. Éste se encarga de controlar los diversos procesos del cuerpo. Entre sus funciones está el procesamiento de los datos de los órganos sensoriales que generan la información de los sentidos: visión, tacto, olfato, gusto y oído. Adicionalmente el cerebro es el encargado de procesar funciones de alta complejidad (emociones, memoria, pensamientos, lenguaje).

El cerebro tienes funciones vitales en el control de movimientos de los órganos; él genera incluso prioridades en funciones corporales homeostáticas, tales como la presión sanguínea, el ritmo cardiaco, la temperatura y el balance de fluidos. A su vez, permite el control voluntario de los miembros (función motora) y realiza un análisis kinestésico de los mismos.

Cuando el cerebro se encuentra funcionando, produce una serie de señales eléctricas. Estas señales se generan según la zona de procesamiento encargada de la función en específico y con unas características particulares la cuales pueden ser cuantificadas, tales como la amplitud y la frecuencia. El dispositivo Emotiv-EPOC (figura 1) aprovecha estas características con el fin de procesar la información proveniente de estas neuroseñales para identificar algunos procesos, tales como emociones, análisis cognitivo y algunas expresiones faciales.

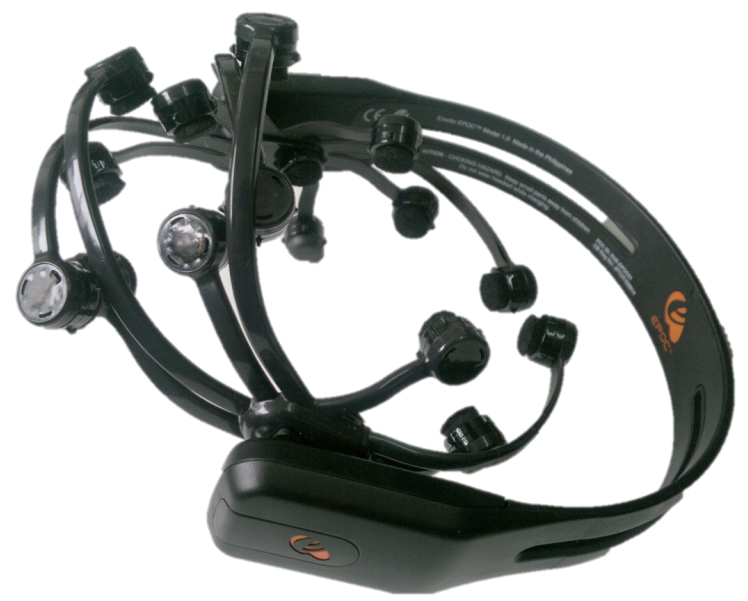

Figura 1. Dispositivo EMOTIV-EPOC 1.0

En la figura 2 se puede apreciar la distribución de los sensores (en la cabeza del usuario) encargados de obtener las neuroseñales. Los sensores se simbolizan por medio de los círculos de color (el verde significa una adecuada captura de las neuroseñales, de lo contrario aparecerá como amarilla cuando la señal posee ruido moderado; café, con ruido significativo; rojo, con ruido crítico y negro cuando la señal no es detectada).
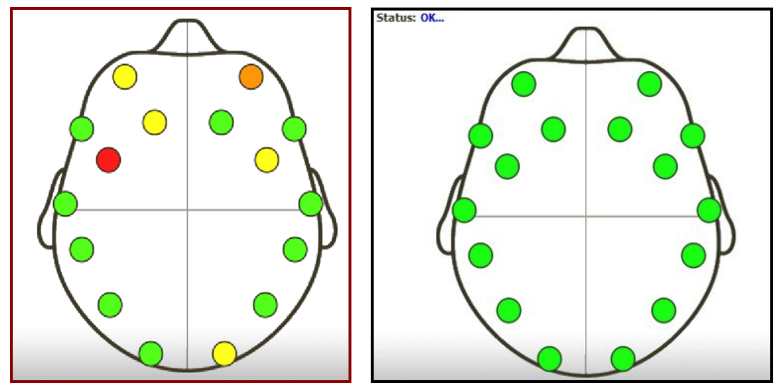

Figura 2. Distribución de los sensores

Los sensores del dispositivo Emotiv-EPOC se encargan de capturar las señales producidas por el cerebro de acuerdo con su localización [14] y de analizarlas con el fin de identificar un proceso en particular (cognitivo, expresivo o emocional). Las ondas capturadas se pueden clasificar en cuatro según su frecuencia: Delta (1-4 Hz), Theta (4-7 Hz), Alfa (7-13 Hz) y Beta $(13-30 \mathrm{~Hz})[15,16]$. En la figura 3 se puede 
apreciar un ejemplo del mapa de actividad cerebral de un usuario cuando está apretando los dientes. Esta acción puede ser fácilmente identificada por medio de las ondas beta (figura 3, parte inferior, derecha) debido a que son generadas en las mismas zonas del cerebro y con una intensidad similar.

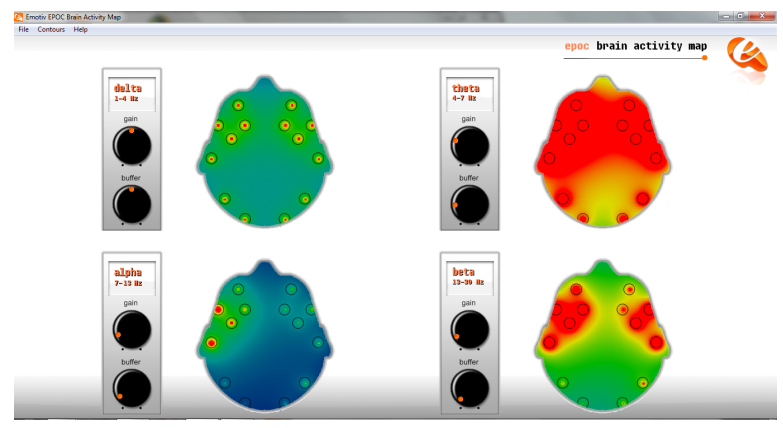

Figura 3. Mapa de actividad cerebral

El dispositivo Emotiv-Epoc cuenta con tres entornos o suites que permiten la identificación de neuroseñales y que se describen en las siguientes subsecciones.

\subsection{Detección de expresiones faciales}

La suite expresiva permite identificar los gestos que realiza una persona (figura 4). Esta herramienta puede ser muy útil en el análisis gestual de los televidentes. Por ejemplo, apretar los dietes al observar que un personaje requiere hacer una fuerza durante la escena, o abrir la boca al ser sorprendido por una imagen, o cuando los niños cierran los ojos al ver sucesos de miedo.
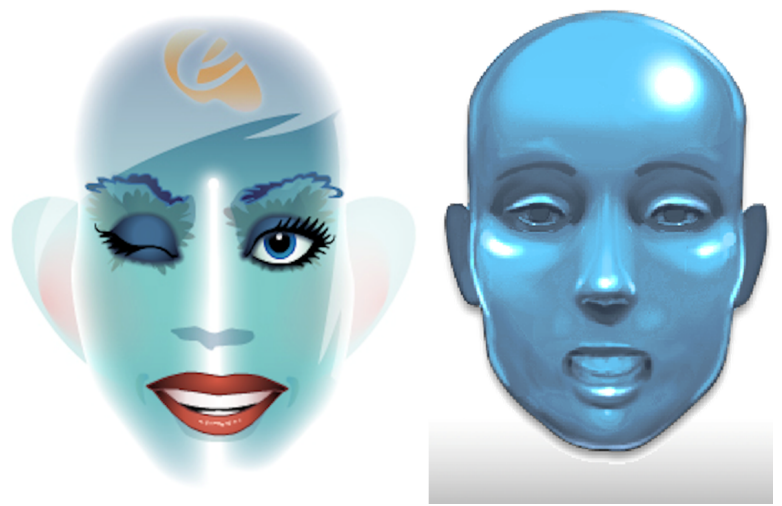

Figura 4. Reconocimiento de gestos
Las expresiones faciales pueden brindar información relevante de la forma como interpretan los televidentes el material audiovisual. Por medio del Emotiv-Epoc se pueden capturar los siguientes gestos: sonrisa, carcajada, dentellado, cerrar los parpados (guiños), movimiento de los ojos, movimientos de las cejas.

\subsection{Detección de emociones}

La suite afectiva proporciona información relevante directamente sobre el estado emocional de los usuarios. Atención o aburrimiento, excitación, calma, frustración y meditación están entre las emociones que se pueden identificar. Estos estados emocionales se pueden estimar a largo y corto plazo. En la figura 5 se aprecia un ejemplo de captura de tres tipos de estados emocionales. Para el análisis de pautas comerciales se recomienda el uso o captura de emociones a corto plazo, debido a que su duración suele ser bastante reducida.

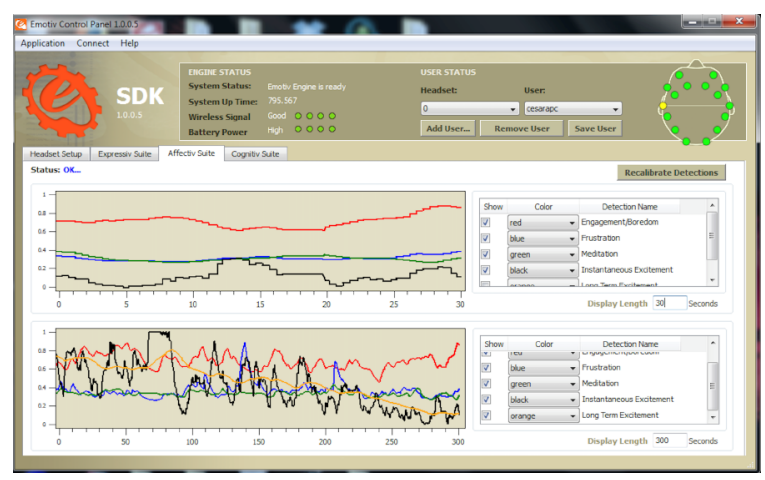

Figura 5. Análisis de emociones

\subsection{Análisis cognitivo}

Si se precisa de un análisis de funciones más complejas, el dispositivo Emotiv-Epoc cuenta con una suite cognitiva que permite identificar algunos tipos de pensamientos. Este procedimiento requiere de un entrenamiento por parte del usuario, por lo que su uso no es frecuente para la toma de muestras experimentales, debido a que implican largos periodos de tiempo.

En la figura 6 se aprecia la interfaz gráfica que permite el entrenamiento del sistema para detectar algunos pensamientos de los usuarios. 
En este ejemplo, el sistema permite detectar cuándo el usuario está pensado en mover o rotar el cubo dibujado, en los tres ejes coordenados. Este mismo método puede ser empleado para detectar colores, letras, imágenes u otros pensamientos del usuario.

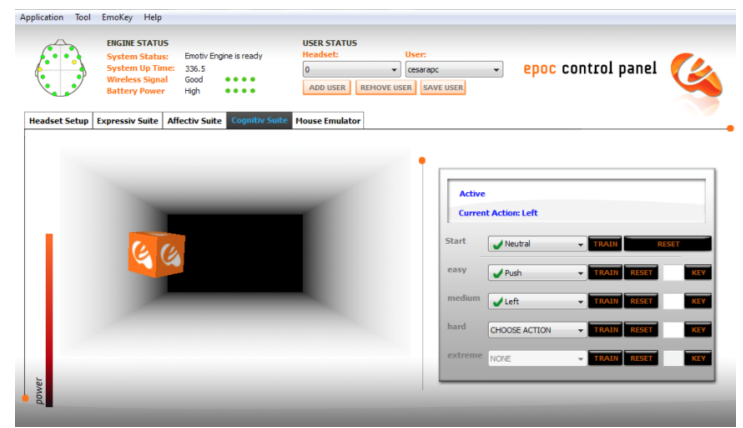

Figura 6. Procesamiento cognitivo

\subsection{Análisis de movimiento por medio de una unidad de medición inercial (IMU)}

Además de las suites afectiva, expresiva y cognitiva, el dispositivo cuenta con una unidad inercial que permite calcular de forma precisa la orientación de la cabeza del usuario. Esta información puede llegar a ser relevante en el análisis de material audiovisual debido a que permitirá detectar si el usuario desvía su atención del televisor, si cabecea debido al sueño o si realiza movimientos bruscos debido a sustos u otras emociones. En la figura 7 se ilustra una aplicación que permite controlar un puntero por medio de los movimientos de la cabeza del usuario.

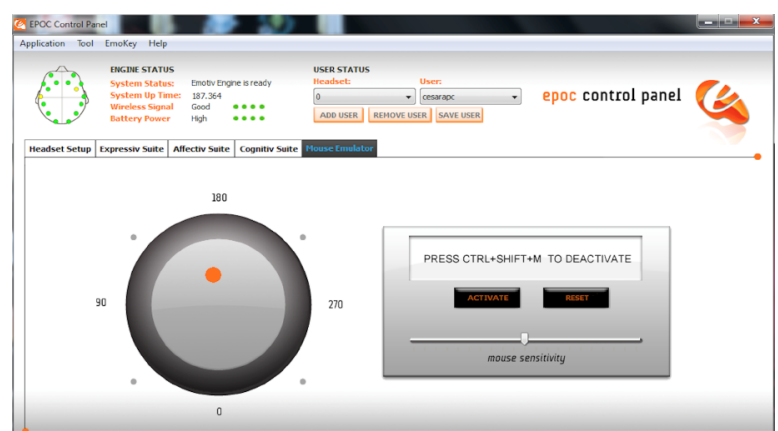

Figura 7. Aplicación de las señales de la IMU para dirigir el puntero del mouse en un PC

\section{ALGORITMOS DE NEUROMARKETING}

En la figura 8 se aprecia la arquitectura del sistema neuromarketing empleado. En primer lugar se reproducen en un televisor las pautas comerciales que se desean analizar, las cuales observa y escucha el usuario. De forma simultánea el dispositivo Emotiv-EPOC envía la neuroseñales vía bluetooth hacia un computador, donde se procesan hasta identificar cada una de acciones o eventos presentados en la sección anterior.

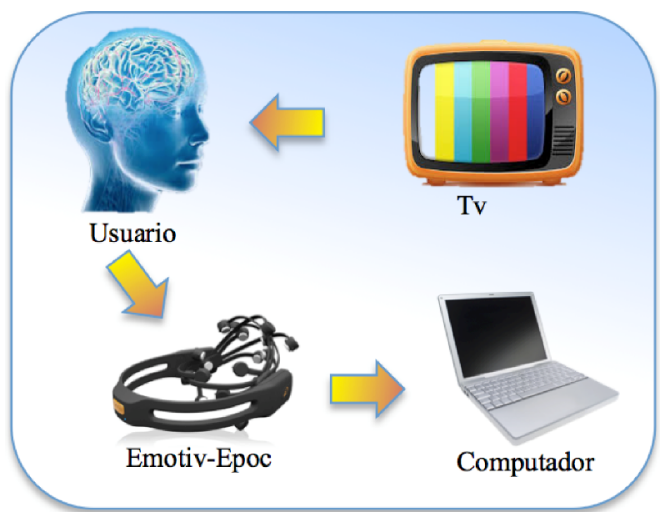

Figura 8. Arquitectura del sistema de neuromarketing

En la figura 9 se aprecian los 7 pasos del procedimiento de neuromarketing propuesto. En el primer paso se hace una adecuación del ambiente de evaluación para que el usuario se encuentre en un entorno sin distracciones (como ventanas, personas, objetos curiosos, ruidos, computadores, radios, etc.). Se prepara el equipo Emotiv-EPOC asegurando la correcta limpieza de sus sensores y se aplica una solución salina, la cual garantiza la conductibilidad entre los sensores y el cuero cabelludo de los usuarios.

Posteriormente se da un tiempo prudente para que el usuario se acostumbre al dispositivo, ello con el objeto de evitar que genere señales erróneas de excitación al sentirse evaluado. Adicionalmente, antes de reproducir cada una de las pautas publicitarias se reproduce una imagen en blanco durante 30 segundos con el fin de neutralizar el estado emocional del televidente. 


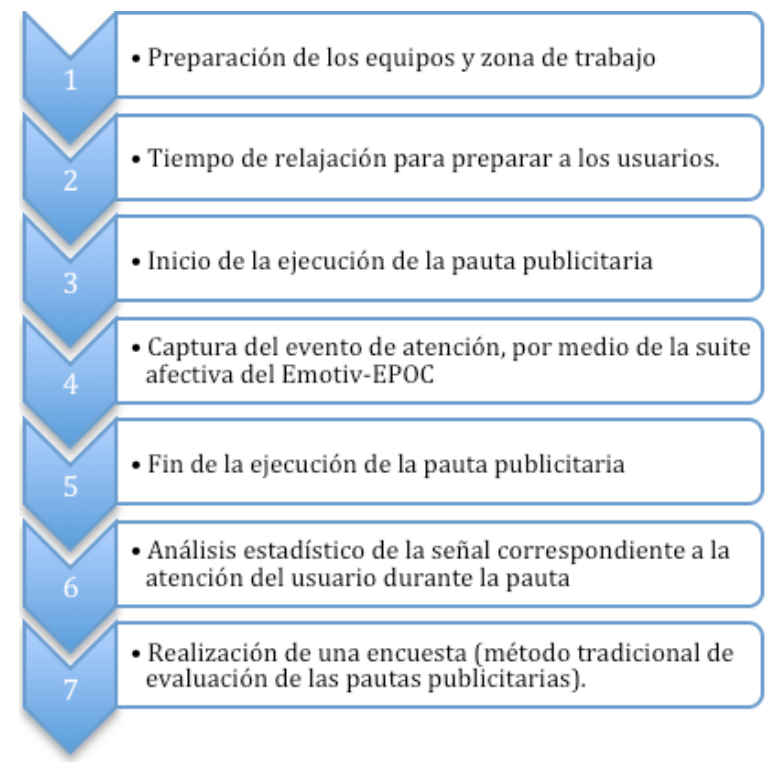

Figura 9. Algoritmo propuesto

El tercer paso consiste en ejecutar el spot publicitario. Cabe aclarar que a un mismo usuario se le proyectan varias pautas publicitarias, mezcladas con material audiovisual, con el fin de acostumbrarle al dispositivo y hacerle olvidar que está siendo evaluado. Varios spots proyectados inicialmente no son tenidos en cuenta para el análisis, debido a los efectos de ansiedad que puede manifestar el usuario al estar sometido al procedimiento.

De forma simultánea con la proyección del material audiovisual, se capturan las neuroseñales por medio del dispositivo Emotiv-EPOC y se hace uso de la suite afectiva para capturar el estado emocional del usuario. De acuerdo con los diversos experimentos realizados, se encontró que la señal correspondiente al evento emocional de atención/desinterés es un buen indicador para caracterizar el comercial.

Una vez se finaliza la proyección del material audiovisual, se procede al análisis visual y estadístico de los resultados obtenidos (señal de atención). Por último se procede a realizar una encuesta al usuario sobre los spots (método tradicional de evaluación) con el fin contrastar los resultados.

\section{RESULTADOS}

Para corroborar el análisis de neuroseñales se realizaron experimentos donde se seleccionaron 12 pautas comerciales relevantes sobre diversos tipos de productos y temas. Los spots poseen diferentes características que causan diversos efectos sobre los televidentes, tales como risa, tristeza, excitación, alegría, patriotismo, entre otros. De igual forma, con el fin de analizar y evidenciar algunos efectos netamente visuales, se usaron algunas pautas mudas y otras que fueron famosas en otros países (por ejemplo, en países asiáticos), de las cuales los usuarios no pudieran comprendieran su lenguaje verbal, pero sí pudieran ser afectados por algunos sonidos como risas, gritos, aplausos, melodías, etc.

En este artículo se presenta el análisis de tres tipos relevantes de comerciales, con los cuales se quería contrastar el nivel de efectividad de las encuestas frente al análisis de neuroseñales de los usuarios. Cabe recalcar que estos tres comerciales estaban mezclados dentro de las doce pautas mencionadas, con el fin de evitar que los televidentes los identificaran. De igual forma, este número de spots hacía que los usuarios se acostumbraran al dispositivo de captura de neuroseñales, y esto evitó o disminuyó los efectos de ansiedad al sentirse examinados. Así mismo se evitó poner los comerciales de análisis en los últimos tres puestos para evadir los posibles efectos de cansancio. Previamente a cada una de las pautas se proyectaba la pantalla en blanco durante 30 segundos con el fin darle tiempo a los usuarios de normalizar sus emociones y anular los efectos residuales que dejaron los spots anteriores.

Con el fin de no involucrar el buen nombre de las compañías involucradas en la publicidad, no se mencionarán los productos a los cuales hacía referencia; sólo se mencionarán algunos de los elementos o técnicas publicitarias empleadas.

Pauta 1: ha sobresalido por su alta aceptación en un país asiático, es muy conmovedor; a pe- 
sar de estar en otro idioma, tiene subtítulos en español y una duración de 157 segundos (comparativamente larga); el objetivo del promotor es mejorar las relaciones intrafamiliares (pausa gubernamental).

Pauta 2: tiene poca aceptación, emplea pocas técnicas de marketing, los autores mencionan que la principal característica es hacer hablar a un bebé con una voz sombría para informarle a sus padres que no desea los productos que le dan, sino uno en particular (el producto promocionado). Es una pausa de 29,8 segundos (tiempo medio).

Pauta 3: tiene poca aceptación, emplea varias estrategias de marketing, dentro de las cuales están el uso de color rojo intenso, en contraste con fondos en escala de grises, el empleo de elementos artísticos combinados con tecnología, mujeres y hombres atractivos, pequeños elementos de humor, entre otros. Está en otro idioma, aunque bastante familiar (inglés). El objetivo del comercial es vender servicios de transporte aéreo. Es una pauta de 90,7 segundos (tiempo moderado)

A continuación se muestran los resultados obtenidos de las neuroseñales usando la suite emotiva del dispositivo Emotiv-EPOC. Los resultados fueron muy similares en los usuarios examinados, sin embargo se presenta un análisis comparativo del comportamiento para un mismo usuario frente a las tres pautas publicitarias mencionadas, con el fin evitar posibles efectos externos, como lo puede ser la edad, el sexo, las condiciones de estrés entre otros.

En la figura 10 se puede apreciar la curva de nivel de atención para el primer spot publicitario. Se puede valorar que el usuario tuvo un alto nivel de atención.

La figura 11 presenta el nivel de atención para la segunda publicidad, donde se resalta un decrecimiento significativo del nivel de atención.

En la figura 12 se puede apreciar el comportamiento de la atención del televidente. Se destaca una elevada fluctuación, sin embargo los niveles de atención son relativamente altos.

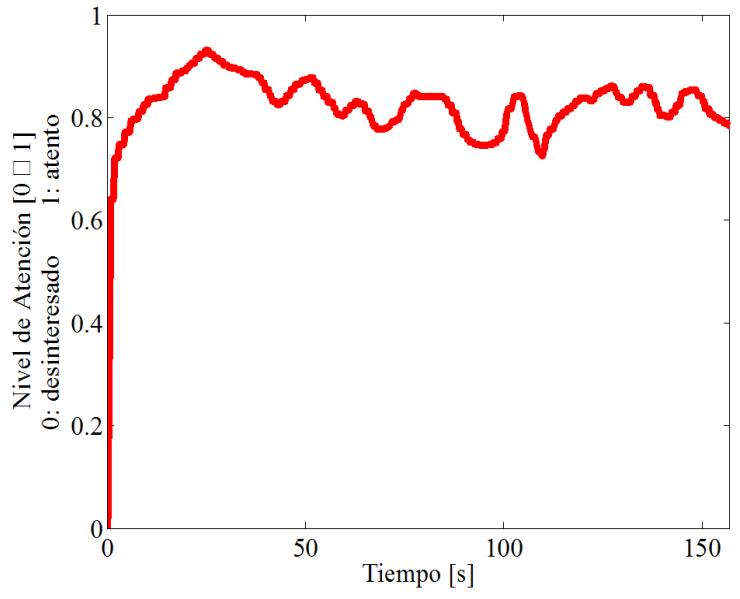

Figura 10. Publicidad 1 - alta aceptación

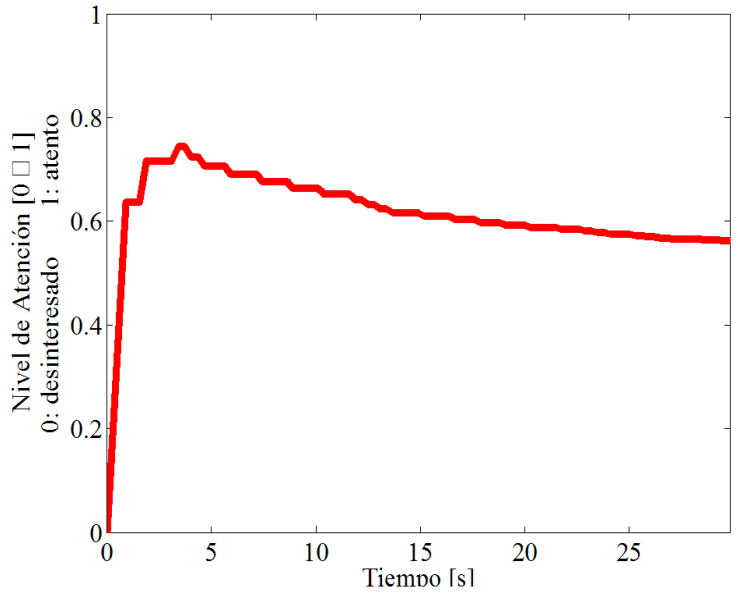

Figura 11. Publicidad 2 - menor aceptación

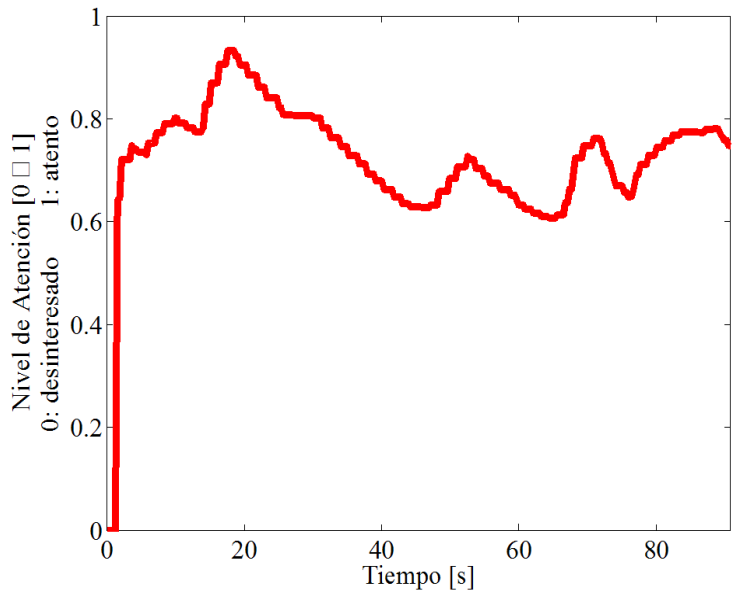

Figura 12. Publicidad 3 - aplicación de varias estrategias de marketing 
Estos resultados son bastante interesantes al ser evaluados y contrastados con las encuestas realizadas. Por ejemplo, el nivel de atención inicial para todas las pruebas resultó moderadamente alto, dado que ninguno de los comerciales había sido visto con antelación por el televidente. Una de las peticiones que se le hacía a los televidentes era ordenar de mayor a menor los doce comerciales expuestos según su agrado. El usuario exhibido en los resultados manifestó que la publicidad 1 fue la de mayor agrado, le gustó por el tema tratado, la consideró conmovedora y la recomendaría. En cambio, la publicidad 2 la juzgó como simple, a pesar de que le causó curiosidad la forma como hablaba el bebé.

Uno de los resultados más relevantes está en la publicidad tres, hacia la cual el televidente manifestó un bajo nivel de agrado, además de que señaló no entenderla en su totalidad debido al idioma en la cual fue presentado. Sin embargo, según el análisis de neuroseñales se detectó un alto nivel de atención. Esto puede significar que los elementos o técnicas de marketing (característica principal de la pauta) causaron un efecto positivo en cuanto al nivel de atención del usuario a pesar de la duración de la pauta publicitaria.

Tabla 1. Indicadores estadísticos de las pruebas realizadas

\begin{tabular}{|c|c|c|c|}
\hline Publicidad & Tipo 1 & Tipo 2 & Tipo 3 \\
\hline Media & 0,8264 & 0,6200 & 0,7283 \\
\hline Mediana & 0,8318 & 0,6099 & 0,7342 \\
\hline $\begin{array}{c}\text { Desviación } \\
\text { Estándar }\end{array}$ & 0,0587 & 0,0817 & 0,1072 \\
\hline Varianza & 0,0034 & 0,0067 & 0,0115 \\
\hline
\end{tabular}

En la tabla 1 se presentan algunos índices estadísticos de las pruebas realizadas, tales como la media, la mediana, la desviación estándar y la varianza. Se puede apreciar un nivel de atención medio superior al $80 \%$ para la primera prueba, cercano al $60 \%$ para la segunda prueba y superior al $70 \%$ para la tercera. En la figura 13 se pueden observar gráficamente estos resultados paramétricos.

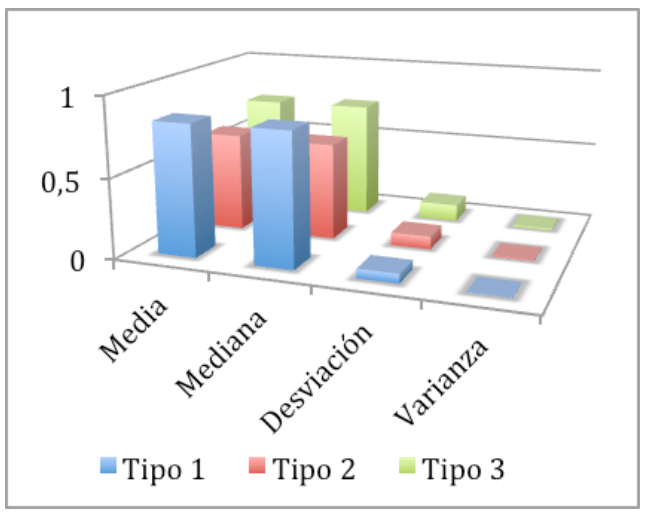

Figura 13. Diagrama de barras de los indicadores estadísticos de las pruebas realizadas

En cuanto a marketing, cabe resaltar que la mala publicidad (comerciales aburridos, algunos errores distintivos) puede generar buenos resultados, dado que se logra el objetivo de crear un elemento vinculante con el usuario para hacerle recordar el producto. Lo que no se puede permitir es que el usuario se lleve una mala imagen del producto.

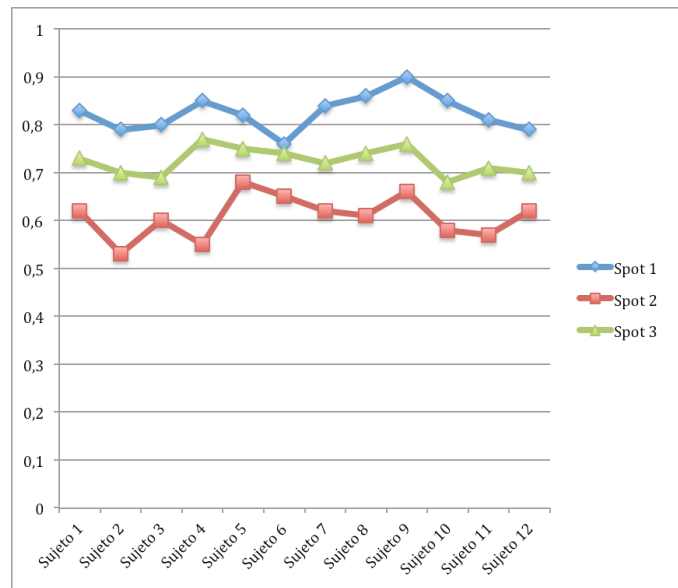

Figura 14. Nivel medio de atención de los usuarios en los tres spots

En la figura 14 se ilustran los resultados correspondientes a los doce usuarios adultos que realizaron el experimento. En estas pruebas participaron 6 usuarios profesionales (entre 25 y 40 años) y 6 estudiantes universitarios (entre 20 y 23 años). Se puede observar una semejanza en los datos obtenidos para los doce sujetos en cada uno de los spots, lo que indica la aplicabilidad del sistema. Cabe recalcar que para estos 
experimentos se tomó una muestra con participantes de nivel de escolaridad similar, con el fin de verificar la concordancia de los datos, sin embargo el sistema es apto para cualquier tipo de usuario, sin importar el sexo, la edad o el nivel de escolaridad. Esto dependerá solamente del tipo de población a la cual vaya ser dirigido o evaluado el spot.

\section{CONCLUSIONES}

Dados los resultados expuestos se puede evidenciar que el análisis de neuroseñales es una herramienta útil en captura del nivel de atención de los usuarios, la cual puede ser utilizada para mejorar la realización, comparación y evaluación de las pautas comerciales. De igual forma se propone para trabajos futuros fusionar la información de los niveles de atención con el análisis de los niveles de frustración/excitación, al igual que el análisis de expresiones

\section{Referencias}

[1] L. M. Keefe, "What Is the Meaning of Marketing?," Marketing News, Vol. 38, No. 15. pp. 17-18, Sept., 2004

[2] $\mathrm{H} . \mathrm{Fu}$ "The father of modern marketing: Philip Kotler Marketing Digest," China Press, ISBN. 780106187X, Vol. 1, Enero. 2000.

[3] M. Soleymani, G. Chanel, J. J. M. Kierkels, and T. Pun, "Affective Characterization of Movie Scenes Based on Multimedia Content Analysis and User's Physiological Emotional Responses," ISM 2008. 10th IEEE International Symposium on Multimedia, pp. 228- 235, Dec. 2008.

[4] K. Yaomanee, S. Pan-ngum, and P. I. N. Ayuthaya, "Brain signal detection methodology for attention training using minimal EEG channels," 10th International Conference on ICT and Knowledge Engineering (ICT \& Knowledge Engineering), pp. 84-89, Nov. 2012.

[5] Z. Ren, X. Qi, G. Zhou, and H. Wang, H., "Exploiting the Data Sensitivity of faciales y corporales de los televidentes.

Según las pruebas realizadas, se puede estimar que el dispositivo Emotiv-EPOC sirve como instrumento de evaluación de las técnicas de marketing implementadas en los comerciales, las cuales no siempre son tenidas en cuenta por los televidentes de una forma consiente, por lo cual no siempre son reflejadas de forma directa en los métodos tradicionales de evaluación como encuestas.

Al obtener señales cuantificables, el dispositivo de captura de neuroseñales puede ser usado por periodos largos: para la evaluación de películas, noticieros, documentales, entre otros, y ofrecer resultados de forma continua que superan algunos de los principales problemas de las encuestas, como el olvido de algunas escenas, la falta de caracterización de efectos puntuales, escribir mentiras por contestar rápido, etc.
[6] Y. Chih-Chin \& J. T. Huang, "The Era of Cloud Computer: Thru Bio-detecting and Open-Resources to Achieve the Ubiquitous devices," IEEE International Conference on Consumer Electronics (ICCE), pp. 580 - 583. Jan. 2012.

[7] L. Grimaldi, "Measuring the Efficacy of Advertising Communication with Neuroscience Methods: An Experiment Performed by Telecom Italia," Pulse, IEEE, vol.3, no. 3, pp. 48-52, May 2012.

[8] Ch. Chuan-Yu, L. Yu-Mon, Z. Jun-Ying, "Physiological Angry Emotion Detection Using Support Vector Regression," 15th International Conference on NetworkBased Information Systems (NBiS), pp. 592-596, Sep. 2012.

[9] H. Touyama, "Post-saccadic event related potential toward a new technique for information retrieval," IEEE International Conference on Systems, Man, and 
Cybernetics (SMC), pp. 2939-2942, Oct. 2012.

[10] R. Ohme, \& M. Matukin, "A Small Frog That Makes a Big Difference: Brain Wave Testing of TV Advertisements," Pulse, IEEE, vol. 3, no. 3, pp. 28-33, May 2012.

[11] G. Du-Jian, L. Wang, Q. Zheng, Y. Liu$\mathrm{Li}$, "Neuromarketing: Marketing through Science," International Joint Conference on Service Sciences (IJCSS), pp. 285-289, May 2012.

[12] F. Babiloni, "Consumer Nueroscience: A New Area of Study for Biomedical Engineers," Pulse, IEEE, vol. 3, no. 3, pp. 21-23, May 2012.

[13] M. Kh. Hazrati, U. G. Hofmann, "Avatar navigation in Second Life using brain signals," IEEE 8th International Symposium on Intelligent Signal Processing (WISP), pp. 1-7, Sept. 2013
[14] D. Wijayasekara, \& M. Manic, "Human machine interaction via brain activity monitoring," 6th International Conference on Human System Interaction (HSI), pp. 103-109, June 2013.

[15] G. Vecchiato, et al., "Enhance of theta EEG spectral activity related to the memorization of commercial advertisings in Chinese and Italian subjects," 4th International Conference on Biomedical Engineering and Informatics (BMEI), vol. 3, pp. 1491-1494, Oct. 2011.

[16] M. Murugappan, S. Murugappan, Balaganapathy, C. Gerard, "Wireless EEG signals based Neuromarketing system using Fast Fourier Transform (FFT)," IEEE 10th International Colloquium on Signal Processing \& its Applications (CSPA), pp. 25-30, March 2014. 